\title{
Imaging Damage in Steel Using a Diamond Magnetometer
}

\author{
L. Q. Zhou $\odot,{ }^{1}$ R. L. Patel, ${ }^{1,2}$ A. C. Frangeskou, ${ }^{1, \dagger}$ A. Nikitin $\odot,{ }^{1,+}$ B. L. Green $\odot,{ }^{1}$ B. G. Breeze, ${ }^{1,3}$ \\ S. Onoda $\odot,{ }^{4}$ J. Isoya, ${ }^{5}$ and G. W. Morley ${ }^{1,2, *}$ \\ ${ }^{1}$ Department of Physics, University of Warwick, Gibbet Hill Road, Coventry CV4 7AL, United Kingdom \\ ${ }^{2}$ EPSRC Centre for Doctoral Training in Diamond Science and Technology, University of Warwick, Coventry CV4 \\ 7AL, United Kingdom \\ ${ }^{3}$ Spectroscopy RTP, University of Warwick, Gibbet Hill Road, Coventry CV4 7AL, United Kingdom \\ ${ }^{4}$ National Institutes for Quantum and Radiological Science and Technology, 1233 Watanuki, Takasaki, Gunma \\ 370-1292, Japan \\ ${ }^{5}$ Faculty of Pure and Applied Sciences, University of Tsukuba, Tsukuba 305 -8573, Japan
}

(Received 22 September 2020; revised 20 November 2020; accepted 7 December 2020; published 5 February 2021)

\begin{abstract}
We demonstrate a simple, robust, and contactless method for nondestructive testing of magnetic materials such as steel. This uses a fiber-coupled magnetic sensor based on nitrogen-vacancy centers (NVCs) in diamond without magnetic shielding. Previous NVC magnetometry has sought a homogeneous bias magnetic field on the diamond to improve the sensitivity. In contrast, here we show that the spatial resolution for imaging is improved by applying an inhomogeneous magnetic field to the steel even though this leads to an inhomogeneous magnetic field on the diamond. Structural damage in the steel distorts the inhomogeneous magnetic field and by detecting this distortion we reconstruct the damage profile through quantifying the shifts in the NVC Zeeman splitting. With a 1-mm magnet as the source of our inhomogeneous magnetic field, we achieve a high spatial resolution of $1 \mathrm{~mm}$ in the plane parallel and $0.1 \mathrm{~mm}$ in the direction perpendicular to the surface of the steel. This works even when the steel is covered by a nonmagnetic material. The lift-off distance of our sensor head from the surface of 316 stainless steel is up to $3 \mathrm{~mm}$.
\end{abstract}

DOI: 10.1103/PhysRevApplied.15.024015

\section{INTRODUCTION}

The optically detected magnetic resonance (ODMR) of nitrogen-vacancy centers (NVCs) in diamond can be used as a magnetic sensor [1,2]. Key strengths of NVC magnetometry are the high dynamic range [3], operation over wide temperature ranges [4], suitability for highradiation environments [5], and chemical inertness. The property of ODMR allows nanoscale-resolution magnetometry, when employing single centers [6-8]. Conversely, an ensemble of NVCs allows for higher sensitivities at the expense of the spatial resolution [2,9-11], with high sensitivities achieved for both dc [11-16] and ac frequencies

\footnotetext{
*Gavin.Morley@warwick.ac.uk

${ }^{\dagger}$ Current address: Lightbox Jewelry, Orion House, 5 Upper St. Martin's Lane, London WC2H 9EA, United Kingdom.

$\ddagger$ Current address: School of Engineering, University of Warwick, Coventry CV4 7AL, United Kingdom.

Published by the American Physical Society under the terms of the Creative Commons Attribution 4.0 International license. Further distribution of this work must maintain attribution to the author(s) and the published article's title, journal citation, and DOI.
}

[17,18]. A range of applications have been demonstrated, from single-neuron action-potential detection [13] to eddycurrent-induced magnetic field detection of conductive samples [19] for material analysis.

The identification of structural defects through the use of magnetic flux leakage (MFL) is a nondestructive testing technique that is among the most-used methods for yielding information about the nature of unknown defects in magnetic materials [20-28]. MFL measurements involve magnetically saturating the target material. If there is no damage, the magnetic flux lines are unperturbed, but if there is a flaw, magnetic flux will leak out of the material. MFL measurements have found applications in industries where the corrosion of magnetic material, such as steel, will eventually lead to significant material loss and, in particular, have been heavily utilized by the oil and gas industries in pipeline inspection gauges to minimize the need for costly excavations [29-37]. Several different magnetic field sensors are employed in industry, with the key technologies being induction coils, Hall-probe sensors and fluxgates, with each offering distinct advantages and disadvantages [30]. Hall sensors in particular have found great usage for MFL measurements due to their low cost $[30,38,39]$; however, these sensors suffer from voltage 
drift, even in the absence of a magnetic field, and thus require compensation and offer limited sensitivity in comparison to other sensors [30,38]. In addition, to effectively utilize the techniques of MFL, the magnetic material under inspection must undergo magnetic saturation [27,28,30]. This requirement sometimes makes it difficult to utilize the technique in the field.

Two quantum systems have been used recently for imaging structural damage in metals $[19,40]$. The first used an atomic vapor cell to achieve a spatial resolution of 0.1 $\mathrm{mm}$ in the direction perpendicular to the surface of the steel [40]. However, this required the use of a sensitive commercial fluxgate magnetometer and 1-m electromagnet coils to null the background magnetic field. Chatzidrosos et al. [19] have demonstrated an NVC magnetometer design without microwaves to remove the problem of the ODMR microwaves interfering with the conductive materials under study. This required a relatively high external bias magnetic field of $102.4 \mathrm{mT}$, supplied by a large water-cooled electromagnet.

Our sensor is based on ODMR of an NVC ensemble, but the use of microwave excitation means that we only need to apply a low bias magnetic field from two permanent magnets. Our sensor-head design prevents microwave leakage with a small Faraday shield and does not use any compensation coils. Under our configuration, we detect magnetic-flux-profile perturbations that arise in the permanent-magnetic-bias fields that are used to induce Zeeman splitting of the NVC; a simulated twodimensional (2D) magnetic flux profile of the setup is shown in Appendix G. These perturbations occur when the magnetic properties of the material are changed, such as by structural defects due to corrosion. This method of flux detection allows for reconstruction of the profile of defects in magnetic materials such as common steels. Furthermore, as the techniques employed do not require magnetic saturation, we thus avoid some of the limitations inherent to magnetic-flux-leakage measurements. We are still able to provide a reconstruction of the examined defects without the need for magnetic shielding. Our fiber-coupled design with a small sensor head increases the flexibility for practical applications. The large lift-off of up to $3 \mathrm{~mm}$ allows this sensor to examine magnetic materials directly even when coated with a thick nonmagnetic layer.

\section{EXPERIMENTAL DETAILS}

We have previously achieved a sensitivity of $310 \pm$ $20 \mathrm{pT} / \sqrt{\mathrm{Hz}}$ in the frequency range of $10-150 \mathrm{~Hz}$ using an isotopically purified ${ }^{12} \mathrm{C}$ diamond with a similar configuration [41]. However, this configuration had poor spatial resolution for imaging. Here, we solve this problem by introducing a $1-\mathrm{mm}$ permanent magnet, $2 \mathrm{~mm}$ from the diamond. While improving the spatial resolution (due to the steel experiencing an inhomogeneous bias field), the resulting inhomogeneous bias field on the diamond impairs the magnetic sensitivity. This means that there is no need to use ${ }^{12} \mathrm{C}$ diamond because our measurements are not limited by the natural abundance of ${ }^{13} \mathrm{C}$ impurities. However, the improvement of the sensitivity is still useful as it allows a higher lift-off from the steel. (a)

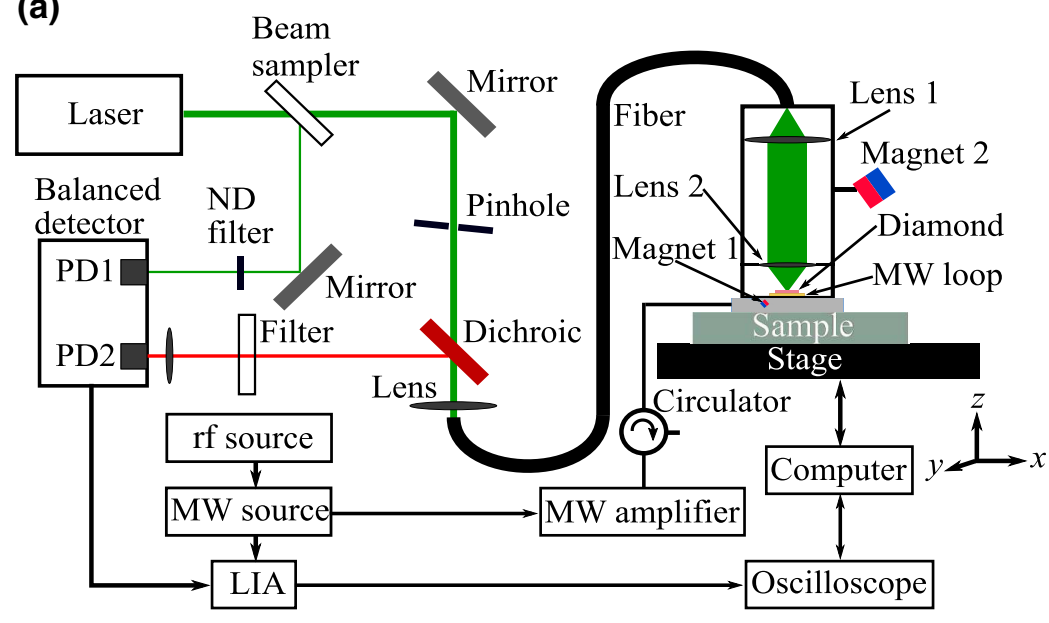

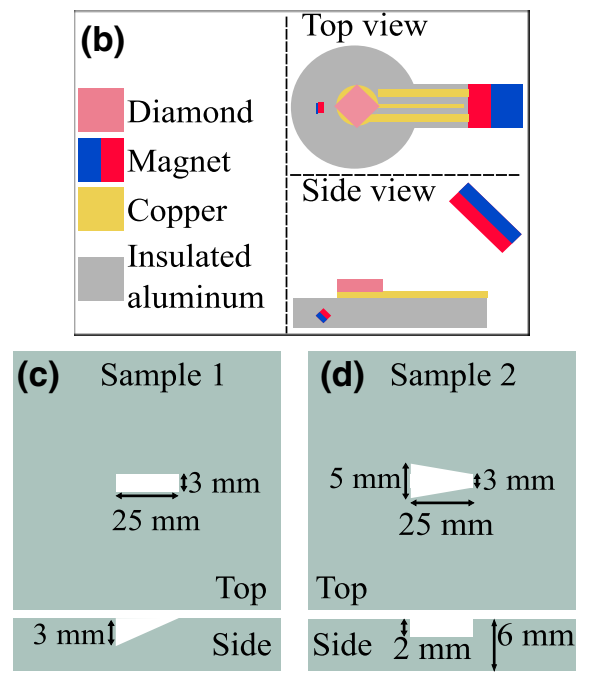

FIG. 1. (a) A schematic of the experimental setup, including the neutral-density (ND) filter, microwave (MW) and radio-frequency (rf) sources, the lock-in amplifier (LIA), and the two photodiodes (PDs) of the balanced detector. The light-gray rectangle containing magnet 1 is the aluminum on which the microwave antenna is printed. (b) A schematic of the microwave antenna used for microwave delivery. A pair of magnets are used to induce Zeeman splitting (the blue and red colors are used to illustrate the differing magnetic poles). (c),(d) The 316-stainless-steel plates used in this work. The plate dimensions are $150 \times 150 \mathrm{~mm}$ : here only a small area where the defects are introduced onto the plates is shown for clarity; the size of the schematics is not to scale. 
The experimental setup is shown in Figs. 1(a) and 1(b); see Appendix B for more details about the experimental setup and Appendix $\mathrm{C}$ for more information about the diamond. Figures 1(c) and 1(d) show the two test samples used; each is made of 316 stainless steel with different slot defects. Sample 1 contains a slot with a width of $3 \mathrm{~mm}$, with a gradient depth from 0 to $3 \mathrm{~mm}$. Conversely, sample 2 has a slot with a fixed depth of $3 \mathrm{~mm}$ and a width ranging from 3 to $5 \mathrm{~mm}$. Both samples are mounted onto the bed of a scanning stage while the sensor head is affixed to the $z$-axis component stage. The scanning stage is used to enable 2D scanning in the $x$ and $y$ axes, with a different liftoff distance in the $z$ axis. All data from the balanced output are digitized using a Zurich MFLI DC-500 kHz lock-in amplifier (LIA), with the microwave frequency modulated.

\section{RESULTS AND DISCUSSION}

The sensitivity of NVC magnetometers is highly dependent on the orientation of the magnetic fields relative to the NVC symmetry axis and the microwave delivery parameters, such as the microwave power, the frequencymodulation amplitude, and the modulation frequency [41, 42]. An NVC ODMR spectrum where the bias magnetic field is aligned along a $\langle 111\rangle$ orientation is shown [see Fig. 2(a)]. A microwave power of $10 \mathrm{~W}$ is used, with a frequency-modulation amplitude of $4.5 \mathrm{MHz}$ and a modulation frequency of $3.0307 \mathrm{kHz}$; these parameters are used for all measurements relating to structural-defect quantification of the stainless-steel samples. The highlighted region at approximately $3 \mathrm{GHz}$ is the region of the ODMR feature where all scanning measurements of the 316-stainless-steel plates are performed. The optimum parameters of operation [see Fig. 2(b)] are found through variation of the microwave power, between $0.2 \mathrm{~W}$ and 10 $\mathrm{W}$, at a fixed frequency-modulation amplitude and modulation frequency. Conversely, the optimum frequencymodulation amplitude is found through variation between $300 \mathrm{kHz}$ and $6 \mathrm{MHz}$, while the microwave power and modulation frequency are fixed. For each parameter, an ODMR spectrum is taken and linear fits applied around the central frequency of the ODMR feature in conjunction with a $1 \mathrm{~s}$ fast Fourier transform (FFT) of the voltage output of the central frequency. The resulting sensitivity is shown in Fig. 2(b), where each sensitivity is the mean of 96 FFTs; the errors are the standard deviation. We report the sensitivity as the average sensitivity from $1 \mathrm{~Hz}$ to $15 \mathrm{~Hz}$. Appendix D compares the sensitivity with and without the small magnet.

The resonance of the NVC shifts when the value of the external magnetic field changes due to Zeeman shifts of the $m_{s}= \pm 1$ energy levels. Changes to the NVC resonance cause a change in the fluorescence and this correspondingly changes the voltage output of the LIA. In
Fig. 2(c), changes to the external magnetic field incident upon the NVC are caused by changes introduced to the distance between the sensor head and the surface of the 316-stainless-steel plates in a damage-free area. The distance of the sensor head from the surface of the ferromagnetic sample is increased and the voltage changes to the LIA output are found (further details concerning the NVC ODMR changes are discussed in Appendix E). The reference point used is indicated by the dashed line in Fig. 2(a): this corresponds to an LIA output value of $0.6 \mathrm{~V}$. Although we could use any value as the reference voltage, provided that it is along the linear region of the NVC ODMR resonance, and it is typical to use the zero crossing point, we choose to use $0.6 \mathrm{~V}$ as the starting reference voltage to make better use of the large dynamic range, because we know that defects in the steel reduce this voltage. If we were to use $0 \mathrm{~V}$ as the reference point, we would only be using half of the dynamic range. A larger dynamic range allows a larger range of lift-off distances to be used. The calibration in Fig. 2(c) is performed upon a blank area of the 316-stainless-steel sample. In this instance, the reference frequency is chosen as the lowest value of the microwave frequency of the highlighted feature in Fig. 2(a). It is evident in Fig. 2(c) that the Zeeman-induced change experienced by the defects is not linearly related to the distance from the steel plate. This is attributed to the dipolar field pattern from the permanent magnets used [43]. The trend of changes to the LIA output during the lift-off process is independent of the sensitivity [see Fig. 2(c)]; the trends are near identical regardless of the microwave parameters used. This is expected as provided that the measurements are in the linear region of the ODMR feature, the differences should be entirely due to the bias magnets and their position relative to the active area of sensing within the diamond. As the differences caused are directly due to magnetic flux distortions, it is entirely possible to use this technique to map out the surface structure of magnetic materials.

The voltage-change response of the magnetic field sensor caused by the surface structure of the steel samples is shown in Fig. 3. The step sizes of the scans in the $x$ and $y$ directions are $1 \mathrm{~mm}$. To prevent interference from the motors of the scanning stage, a dwell time of $1 \mathrm{~s}$ is implemented before the data-acquisition process begins. This leads to a total scanning time of 2-3 s per point and hence to a total scan time of approximately $66 \mathrm{~min}$ for a $20 \times 45 \mathrm{~mm}$ scan. Both defects are mapped out and clearly visible, as shown in Figs. 3(a) and 3(d). The lift-off distance between the surface of the sample and the sensor head from the base of the antenna on the aluminum printed circuit board (PCB) is $0.2 \mathrm{~mm}$ for all scans in both Fig. 3(a) and Fig. 3(d). Using the voltage difference and the calibration performed in Fig. 2(c), it is possible to evaluate differences in the depth of the features. We believe that the 
(a)

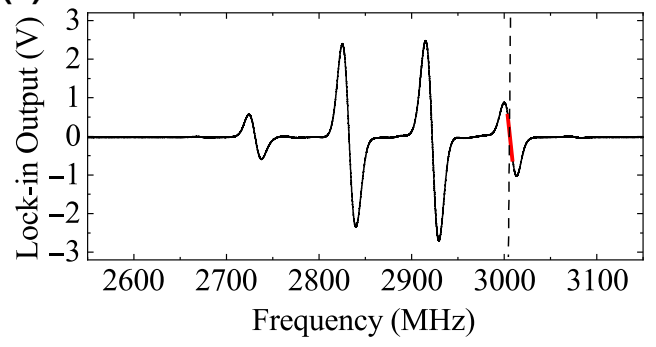

(b) Modulation Amplitude (MHz)
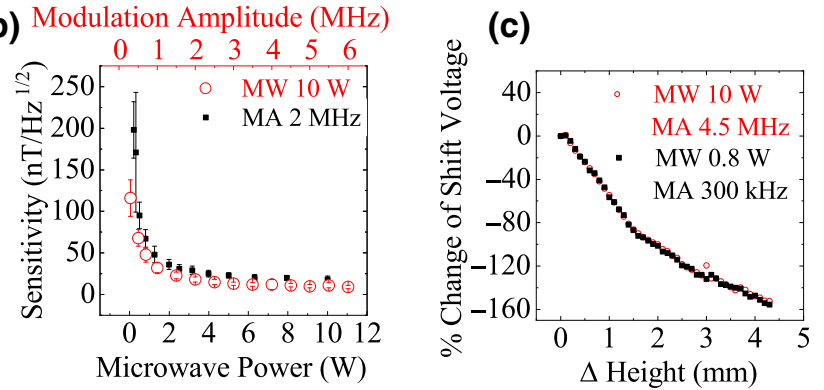

FIG. 2. (a) The ODMR spectrum from the lock-in amplifier (LIA) output. (b) The dependence of the sensitivity on the input microwave power (black square) and the modulation amplitude (MA) (red circle). (c) The changes to the LIA voltage output while monitoring a fixed microwave frequency when the lift-off distance between the sensor head and a damage-free 316-stainless-steel sample is changed is shown. This is taken under two differing sensitivity settings. The lift-off is $0.2 \mathrm{~mm}$ for $\Delta$ Height $=0 \mathrm{~mm}$.

increase in the normalized voltage output in Fig. 3(a) in the top left corner is due to the larger magnet being close to the sample edge.

Figures 3(a) and 3(d) reveal differences in profile and show that damage in a ferromagnetic sample can be differentiated based on its depth and width. To further highlight these differences and confirm the distinction between depth and width, the differences in the profile of the cross sections from Figs. 3(a) and 3(d) are analyzed by performing Lorentzian fits across the width spanned by the defect. Appendix F shows that even though the slot defects have a "top-hat" shape rather than a Lorentzian shape, our data are well fitted by a Lorentzian because of the imperfect spatial resolution of our measurements. It is expected, for a sample where the depth changes but not the width, that the magnitude of the change to the LIA voltage output will increase across the length of the defect but that this change will not be reflected in changes to the full width at half maximum (FWHM), which should remain fairly constant. This is demonstrated by Fig. 3(c), where the FWHM is for the most part constant, while Fig. 3(f) shows that the amplitude increases in an almost linear fashion with increases with the depth of the defect. The rapid change in the shift voltage in Fig. 3(b) is due to the boundary of the defect edge, where the depth change is at a maximum.

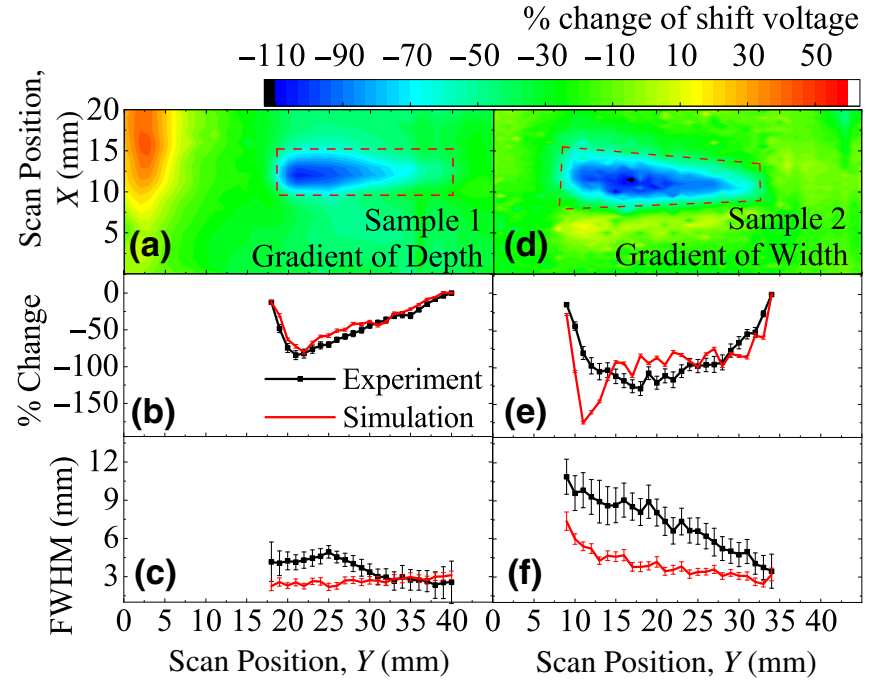

FIG. 3. (a),(d) 2D scan results for steel samples (a) 1 and (d) 2; respectively. The scan dimensions are $45 \times 20 \mathrm{~mm}$. (b),(e) The amplitudes of Lorentzian fits to the cross-section profiles of (a) and (d), respectively. (c),(f) The full width at half maximum (FWHM) of the cross sections of both defects imaged by the sensor, shown for samples 1 and 2, respectively. COMSOL simulations are performed and the results are shown in (b), (c), (e), and (f).

In contrast, it is expected that the magnitude of the change for a sample the depth of which is unchanged will be constant across the length of the defect, while the FWHM will change with a larger width resulting in a larger FWHM value. This is confirmed by Fig. 3(f), which shows a constant increase in the FWHM as the width of the defect increases but relatively constant voltage changes, as shown in Fig. 3(e). COMSOL simulations of the experiment (see Appendix $G$ for details) yield defect cross sections and magnitude signals that are in overall agreement with the experiment. These results are shown in Figs. 3(b), 3(c), 3(e), and 3(f). The steel relative magnetic permeability used is 1.02 in the simulation, as discussed in Appendix G.

Steel corrosion under insulation is an important global problem and we demonstrate that it is possible to map the defects even when they are covered by nonmagnetic materials, as shown in Figs. 4(a)-4(d). The lift-off distance is $3 \mathrm{~mm}$ for all scans in Fig. 4; when compared to those in Fig. 3, the larger lift-off means that the magnetic flux from the smaller magnet is incident over a larger area of the steel. This leads to a worse spatial resolution in the plane parallel to the sample surface: the $x$ and $y$ directions. There are also more spurious signals than in Fig. 3, which will be addressed in future research. On the $z$ axis, we achieve a $0.1 \mathrm{~mm}$ spatial resolution, while for the $x$ and $y$ axes, we reach approximately $1 \mathrm{~mm}$, which we believe is limited by the 1-mm size of the small magnet. The minimum lift-off on the $z$ axis is set by the nominal resolution of the scanning stage on the $z$ axis; increments lower than $0.1 \mathrm{~mm}$ are 


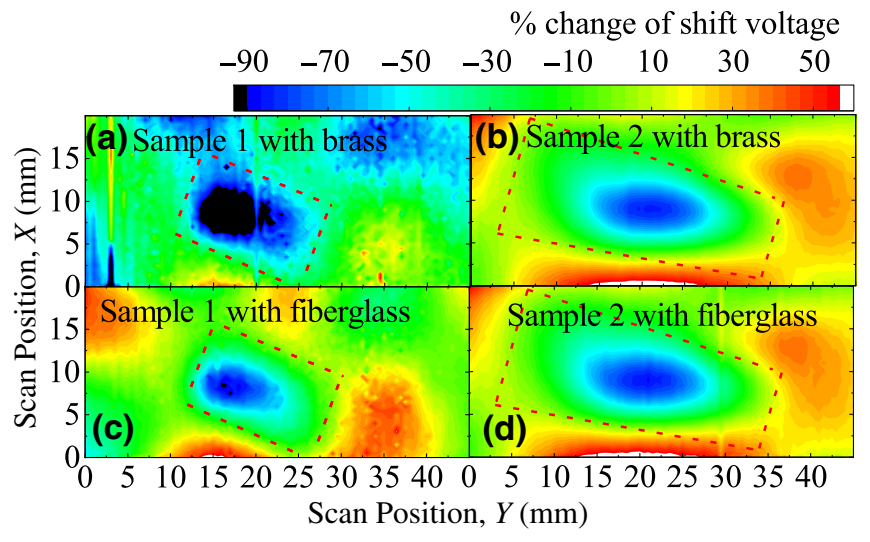

FIG. 4. Two dimensional (2D) scans of samples 1 and 2 when covered with nonmagnetic material covering. The scan dimensions are $45 \times 20 \mathrm{~mm}$. The structural defects present in samples 1 and 2 are encapsulated with the dashed line: (a),(c) are from sample 1; (b),(d) from sample 2. For (a) and (b), the steel is covered with $1.5 \mathrm{~mm}$ of brass, while for (c) and (d), the steel is covered with $2 \mathrm{~mm}$ of fiberglass.

not possible. It may be possible to enhance the spatial resolution either through using a smaller magnet or one that is shaped to have a sharp point facing the steel.

\section{CONCLUSION}

In this work, we demonstrate a method of imaging defects in magnetic materials using a compact sensor based on an ensemble of nitrogen-vacancy color centers in diamond. This device can be used to detect structural defects in magnetic materials and aid in their quantification even when covered with nonmagnetic materials. It could be used to find corrosion under insulation. Reducing the size of the 1-mm-cube bias magnet may improve the spatial resolution of our measurements. Furthermore, as the sensor head is based on diamond, with further improvements to the design, we expect that it would be suitable for operation in radioactive environments [5] and at temperatures of up to $300^{\circ} \mathrm{C}$ [4].

All data used in the production of this work is available online [44].

\section{ACKNOWLEDGMENTS}

We thank Luke Johnson for materials processing, Jeanette Chattaway, Matty Mills, and Lance Fawcett of the Warwick Physics mechanical workshop, and Robert Day and David Greenshield of the Warwick Physics electronics workshop. We are particularly grateful for insightful discussions with Steve Dixon, Jozef Tzock, Zhichao Li, and Rachel Edwards (University of Warwick) concerning nondestructive testing. R.L.P.'s Ph.D. studentship is funded by the Engineering and Physical Sciences Research Council
(EPSRC) Centre for Doctoral Training in Diamond Science and Technology (Grant No. EP/L015315/1). L.Q.Z. is supported by an EPSRC Impact Acceleration Account grant from Warwick Ventures. The use of the Spectroscopy Research Technology Platform facilities at the University of Warwick is acknowledged. B.L.G. is supported by the Royal Academy of Engineering. This work is supported by the UK Hub NQIT (Networked Quantum Information Technologies) and the UK Hub in Quantum Computing and Simulation, part of the UK National Quantum Technologies Programme, with funding from UKRI EPSRC grants (Grants No. EP/M013243/1 and No. EP/T001062/1, respectively). G.W.M. is supported by the Royal Society.

\section{APPENDIX A: NITROGEN VACANCY CENTER MAGNETOMETRY PRINCIPLES}

The NVC in diamond is a spin-1 color center with a zero-field splitting of $2.87 \mathrm{GHz}$. The system is comprised of two spin triplet states, the ground and excited states, ${ }^{3} A_{2}$ and ${ }^{3} E$, respectively, along with two singlet states, ${ }^{1} A_{1}$ and ${ }^{1} E$ (see Fig. 5). The property of ODMR allows spin-state readout through spin-dependent transitions that cause a reduction in the red fluorescence emitted by the NVC when in the $m_{s}= \pm 1$ state compared to the $m_{s}=0$ state [1]. This reduction is associated with transitions through an intersystem crossing to the ${ }^{1} A_{1}$ state and then the ${ }^{1} E$ state. The ${ }^{1} E$ state has a relatively long lifetime [1]. The probability of intersystem crossing is higher for the $m_{s}= \pm 1$ states. Optical excitation using a 532-nm laser allows spin initialization into the ${ }^{3} A_{2} m_{s}=0$ state. These properties allow high signal-to-noise magnetic field detection when a bias magnetic field is applied to the NVC [10].

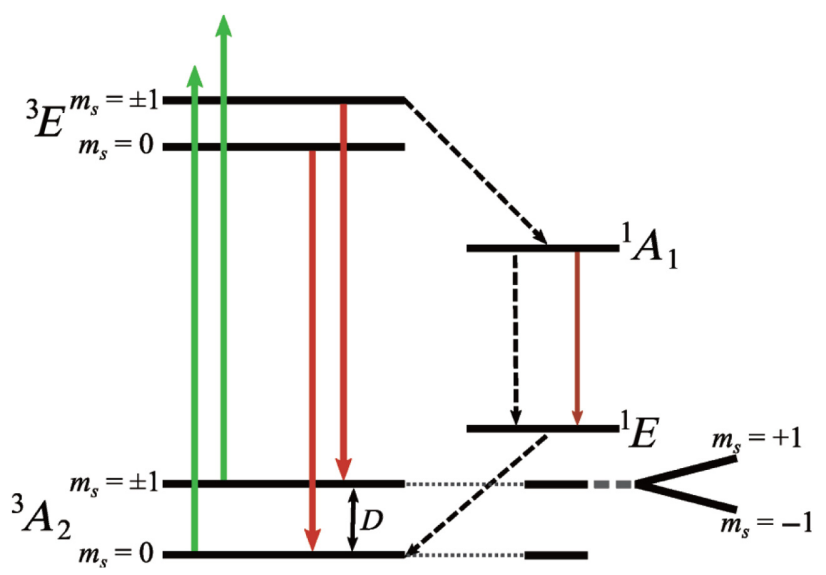

FIG. 5. The energy-level diagram of the negatively charged NVC. The parameter $D$ is approximately $2.87 \mathrm{GHz}$ at room temperature. Nonradiative transitions are indicated by dashed lines. The nondashed transition between the ${ }^{1} A_{1}$ and ${ }^{1} E$ energy levels is the 1042-nm infrared transition. Under no applied bias field, the $m_{s}= \pm 1$ states are degenerate. When a magnetic bias field is applied, the states are Zeeman split. 


\section{APPENDIX B: EXPERIMENTAL DETAIL}

A Laser Quantum 532-nm Gem laser is used to excite fluorescence from the NVC ensemble. The output laser power used is $1 \mathrm{~W}$. A beam sampler (Thorlabs BSF10A) and neutral-density filter (Thorlabs ND10A) are used to pick off $1 \%$ of the laser beam for laser-noise cancellation using a balanced detector (Thorlabs PDB450A). A 650-nm short-pass dichroic mirror (Thorlabs DMSP650) is used to help separate the NVC fluorescence and the laser excitation. For mechanical stability, the diamond is mounted onto a custom-made aluminum microwave delivery antenna board (CIF AAT10), which is affixed to the sensor head. Microwaves are supplied by an Agilent $\mathrm{N} 5172 \mathrm{~B}$ with the carrier wave frequency modulated; this output is mixed with a $2.158 \mathrm{-MHz}$ sine wave generated using an arbitrary function generator (RSPro AFG21005) to simultaneously excite all three ${ }^{14} \mathrm{~N}$ hyperfine resonances. This microwave output is then amplified using a 43-dB gain amplifier (Mini-Circuits ZHL-16W-43-S+) and passed through a coaxial circulator. An approximately 1$\mathrm{mm}$ hole is drilled into the aluminum antenna at an angle of approximately $45^{\circ}$ and a distance from the microwave excitation loop of $5 \mathrm{~mm}$. A 1-mm-cube nickel-plated $(\mathrm{Nd}, \mathrm{Fe}) \mathrm{B}$ magnet (Supermagnete $\mathrm{W}-01-\mathrm{N})$ is inserted into the hole. A second magnet of dimensions $25 \times 25 \times 13$ $\mathrm{mm}$ (Supermagnete Q-25-25-13-N) is used to reduce the strength of the magnet nearest to the diamond to enhance the magnetometer sensitivity; this magnet is housed in a custom three-dimensionally printed holder, which is affixed to the sensor head and enables a $360^{\circ}$ rotation of axes in two dimensions (those parallel to the $x$ and $y$ axes) and orientation control in the axis parallel to the $z$ axis allowing for arbitrary alignment of the magnetic field incident on the NVC ensemble.

The detection of damage in a magnetic material is possible as we monitor the magnetic-flux-profile change of the two magnets. The $1-\mathrm{mm}$-cube magnet is used as a probe that injects magnetic flux into a magnetic material. When the magnetic properties of the material under study changes, this will affect the magnetic flux profile of the two magnets. As the same magnets are also used to induce Zeeman splitting on the NVC ensemble, when there are any changes to the magnetic flux, they can be detected by the NVC ensemble. Due to the small size of the magnet closest to the diamond, the field incident upon the NVC ensemble is highly inhomogeneous. As the small magnet is used as a probe to infer the magnetic properties of a material, the spatial resolution is directly correlated with the area of the sample that is magnetized. In addition, the spatial resolution is also affected by the volume excited by the 532-nm laser. For our excitation area, we measure a beam waist of approximately $40 \mu \mathrm{m}$, with the excitation on the $z$ axis being $0.24 \mathrm{~mm}$ (the thickness of the diamond).

\section{APPENDIX C: DIAMOND MATERIAL PROPERTIES}

The diamond sample used in this work is a (100)oriented high-pressure high-temperature (HPHT) sample, purchased from Element Six, of dimensions $1.83 \times 1.85 \times$ $0.24 \mathrm{~mm}$; this is laser cut from a larger HPHT plate. This diamond is selected as it has a lower nitrogen content than other similar HPHT samples. The diamond is electron irradiated with $2 \mathrm{MeV}$ electrons at a dose of $10^{18} \mathrm{~cm}^{-2}$ at room temperature. The diamond sample is afterward annealed for $4 \mathrm{~h}$ at $400{ }^{\circ} \mathrm{C}$, for $2 \mathrm{~h}$ at $800^{\circ} \mathrm{C}$, and for $2 \mathrm{~h}$ at $1200^{\circ} \mathrm{C}$; at each step, a 1-h ramp is used between the temperatures changes [45]. This multistage annealing processes is performed to encourage NVC formation while minimizing the presence of unwanted impurities. The diamond is mechanically polished on all six sides until an optical-grade quality finish is achieved. The diamond is subsequently cleaned in a sulfuric acid $\left(\mathrm{H}_{2} \mathrm{SO} 4\right)$ and potassium nitrate $\left(\mathrm{KNO}_{3}\right)$ solution for $60 \mathrm{~min}$ and then further cleaned for $20 \mathrm{~min}$ in $\mathrm{H}_{2} \mathrm{SO}_{4}$. The concentration of the crystal defects in the diamond is established though electron paramagnetic resonance (EPR), Fourier-transform infrared spectroscopy (FTIR), and ultraviolet-visible (UV-vis) spectroscopy. The UV-vis data are taken using a Perkin Elmer Lambda 1050 spectrometer equipped with an Oxford Instruments Optistat cryostat. The FTIR data are taken at room temperature using a Perkin Elmer Spectrum GX FT-IR spectrometer. The concentrations measured with EPR are $31 \mathrm{ppm}$ of single substitutional nitrogen and $1.8 \mathrm{ppm}$ of negativelycharged NVC. FTIR measures a concentration of $38 \mathrm{ppm}$ of single substitutional nitrogen and $2.9 \mathrm{ppm}$ of singlypositively-charged substitutional nitrogen. UV-vis measures a concentration of $4.3 \mathrm{ppm}$ of negatively-charged $\mathrm{NVC}$ and $0.4 \mathrm{ppm}$ of neutral NVC. The differences in the NVC concentrations between EPR and UV-vis may be due to charge-transfer effects.

\section{APPENDIX D: MAGNETOMETER SENSITIVITY}

As the steepness of the slope of the LIA derivative output of the ODMR spectra directly relates to the magnetic field sensitivity, the data near the zero crossing point of the outermost (111) resonances are determined by applying linear fits around the zero crossing. This provides a calibration with which to convert units of voltage into those of magnetic field. We then take 160 FFTs when monitoring the zero crossing of the ODMR resonance.

Figure 6(a) shows the ODMR derivative spectrum under the dual-magnet configuration, while Fig. 6(b) shows the ODMR spectrum when using only the $25 \times 25 \times 13 \mathrm{~mm}$ magnet as the bias field for the NVC ensemble. The microwave parameters used for the experimental configuration corresponding to Fig. 6(a) are a microwave power 

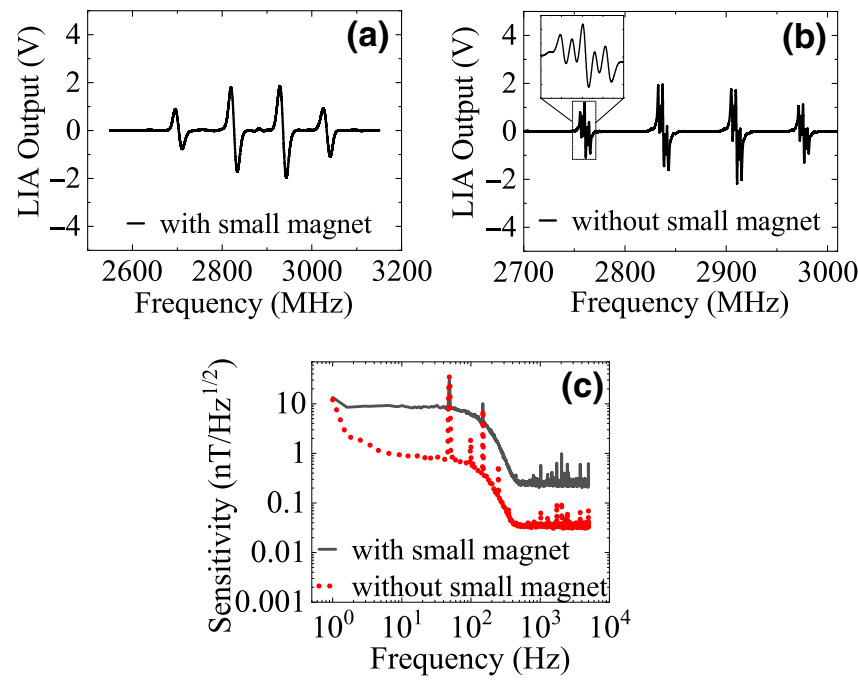

FIG. 6. (a) The ODMR spectrum using the Supermagnete W01-N and Q-25-25-13-N magnets. (b) The ODMR spectrum using only the Supermagnete Q-25-25-13-N. (c) The spectral density corresponding to the configurations in (a) and (b).

of $10 \mathrm{~W}$, a frequency-modulation amplitude of $4500 \mathrm{kHz}$, and a modulation frequency of $3.0307 \mathrm{kHz}$, while the microwave parameters for Fig. 6(b) are a microwave power of $0.2 \mathrm{~W}$, a frequency-modulation amplitude of $350 \mathrm{kHz}$, and a modulation frequency of $3.0307 \mathrm{kHz}$. To quantify the broadening due to using the dual-magnet arrangement, ODMR spectra are taken in the absence of frequency modulation with and without the 1-mm-cube $(\mathrm{Nd}, \mathrm{Fe}) \mathrm{B}$ magnet; the ODMR line width under the dualmagnet arrangement is $13.9 \mathrm{MHz}$, while it is $3.15 \mathrm{MHz}$ when using only the $25 \times 25 \times 13 \mathrm{~mm}$ magnet. For both ODMR spectra, the microwave parameters are optimized to yield the best sensitivity. The microwave frequency is swept in a selected range with a frequency step size of $20 \mathrm{kHz}$. The differences in sensitivity are shown in Fig. 6(c); the sensitivity for the configuration corresponding to Fig. 6(a) is $(9 \pm 1) \mathrm{nT} / \sqrt{\mathrm{Hz}}$, while it is $(0.8 \pm 0.2)$ $\mathrm{nT} / \sqrt{\mathrm{Hz}}$ for the configuration in Fig. 6(b).

\section{APPENDIX E: ODMR SPECTRA UNDER DIFFERENT CONDITIONS}

Figures 7(a) and 7(b) show ODMR spectra taken with and without a 316-stainless-steel sample under the sensor head. Figures 7(c) and 7(d) show the ODMR spectra when the steel sample is under inspection with different lift-off distances; the lift-off distances are between a range of $1-4 \mathrm{~mm}$ and $1-\mathrm{mm}$ increments are used. Figures 7(e) and 7(f) show the ODMR spectra when the sensor head is placed at different positions above the gradient-depth sample. To highlight the resonance shifts, the outermost NVC resonance is shown in Figs. 7(b), 7(d), and 7(f), these correspond, respectively, to the dashed areas in Figs. 7(a),
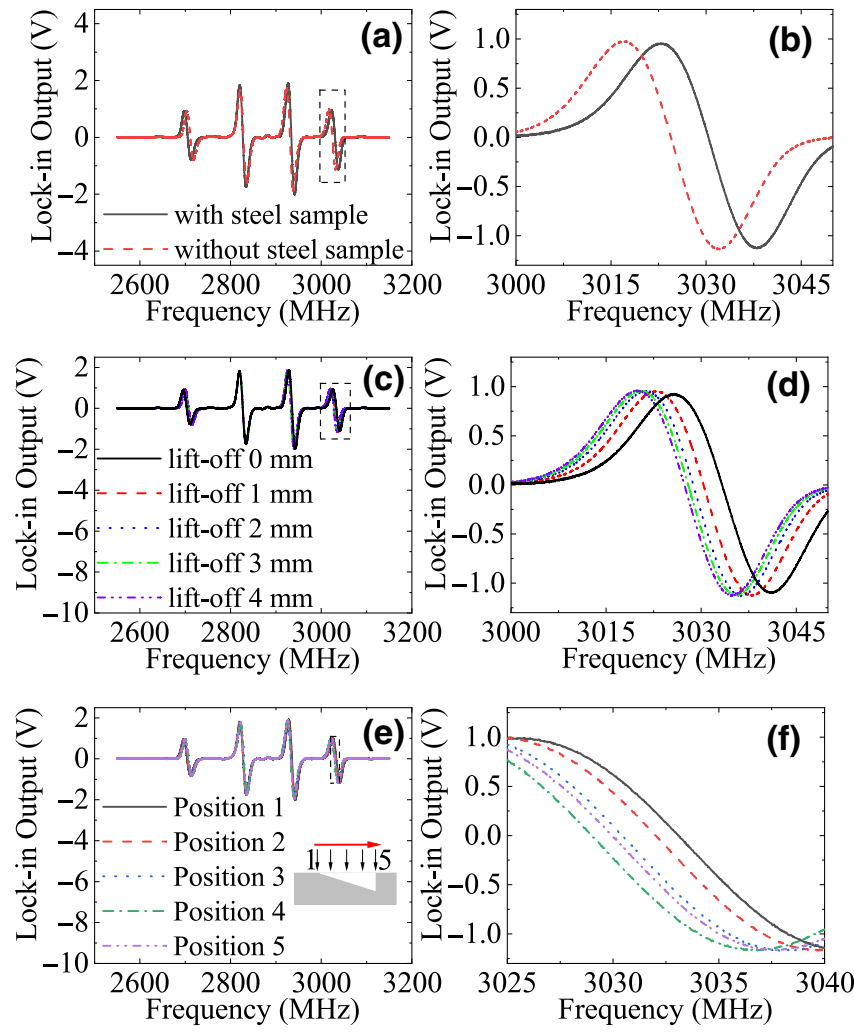

FIG. 7. ODMR spectra in different conditions. (a) Taken with and without the 316-stainless-steel sample. (b) With a lift-off from the steel from $1 \mathrm{~mm}$ to $4 \mathrm{~mm}$. (c) For various positions along the length of the gradient-depth steel sample. (b),(d),(f) These spectra correspond to the dashed regions in (a), (c), and (e) respectively: these are of the outermost right-hand-side NVC resonance.

7(c), and 7(e). As is expected, the NVC ODMR resonances shift correspondingly to the magnetic-flux-profile changes over the NVC ensemble, which is caused by changing magnetic properties near the sensor head.

\section{APPENDIX F: SCAN DATA PROFILE}

In Fig. 8, the scan results of the gradient-depth and gradient-width samples are shown along with the crosssection profile. When considering only the extent of the percentage change shift (relative to our $0.6-\mathrm{V}$ reference) in the LIA output, we do see differences in the profiles for the gradient-depth and gradient-width samples [see Figs. 8(b) and 8(e)]; the differences in the depth are much clearer than that of the width. To highlight the changes in the width, we perform Lorentzian and Gaussian fits [19], as these fit the data even though the slot defect does not have this shape. It is found in our analysis that there is no distinct difference in the obtained FWHM if we use a Lorentzian or a Gaussian profile for the fits and thus we show only the Lorentzian profiles. 


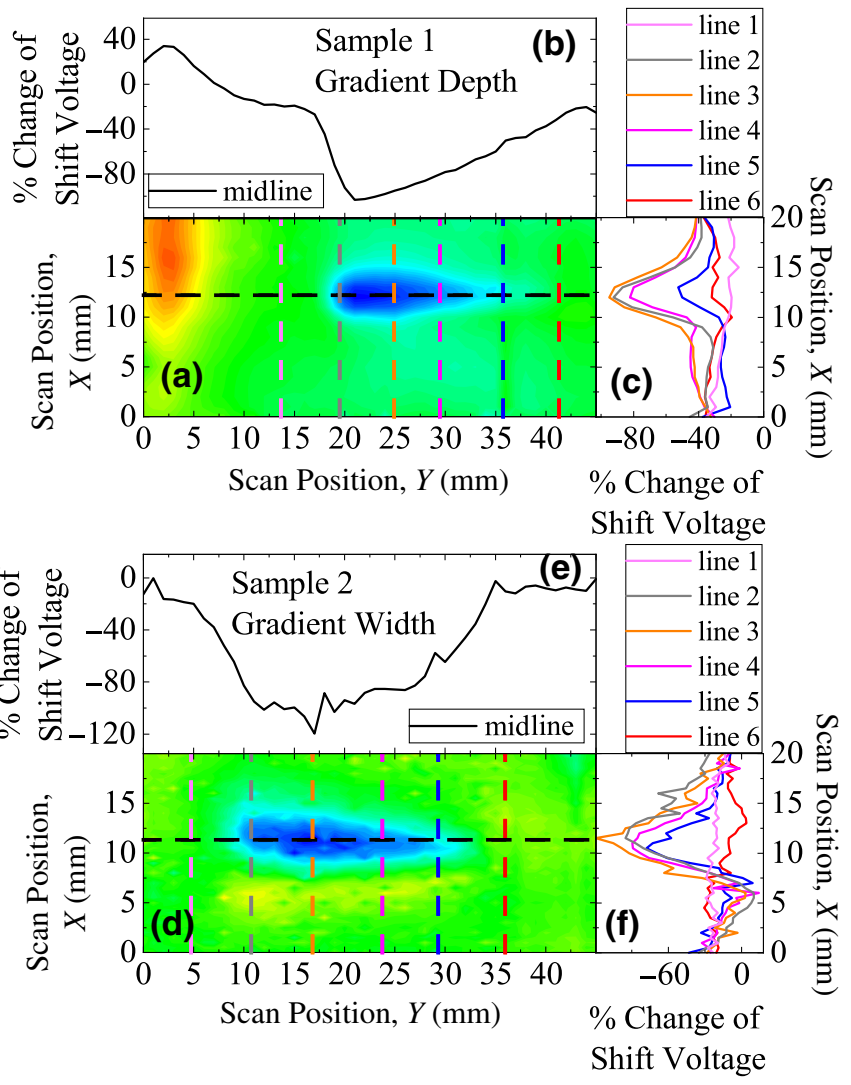

FIG. 8. (a),(d) The 2D scan images of the gradient-width and gradient-depth samples. (b),(e) The percentage changes of the LIA output voltage taken along the lines shown in (a) and (d), which are parallel to the $y$ axis. (c),(f) The percentage changes of the LIA output voltage taken from six lines that are parallel to the $x$ axis in (a) and (d); four of the six lines are taken along the damage area (lines 2-5), while two are taken along areas where no damage is present (lines 1 and 6).

\section{APPENDIX G: FINITE-ELEMENT MODELING SIMULATIONS}

All simulations are performed using the commercial software COMSOL MULTIPHYSICS in the "Magnetic field, No current" mode. A 2D magnetic flux profile simulated using COMSOL is shown in Fig. 9. The simulations of the damage in steel were three dimensional with the geometry of the samples and defects corresponding to those listed in the main text as shown in Fig. 10. The magnet grades used for the simulation are the N45 magnet, for the 1-mmcube magnet, while the larger magnet has a magnet grade of N42; both magnets have a relative magnetic field permeability of 1.05 . The step sizes for Figs. 11(a) and 11(b) are $0.25 \mathrm{~mm}$ along the $x$ axis and $1 \mathrm{~mm}$ along the $y$ axis. The relative permeability of the 316 stainless steel is set to 1.02 .

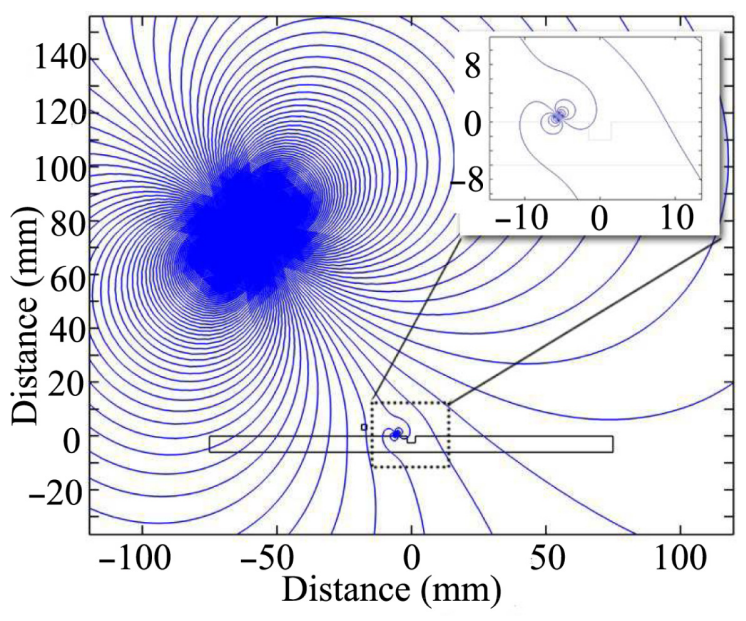

FIG. 9. The magnetic flux profile of a pair of bias fields is simulated using COMSOL. The inset shows an enlargement of the region inside the dotted box including the small magnet and the defect in the steel.

\section{APPENDIX H: DYNAMIC RANGE}

The dynamic range referred to in the main text is the extent of the linear region of the lock-in amplifier derivative signal of the NVC ODMR.

It is desirable to have a high dynamic range as the measurements using the NVC can only be performed when monitoring an NVC resonance. Application of a large magnetic field will shift the resonance such that it is no longer possible to gain any useful information regarding the nature of the defects in a magnetic material. In addition, having a high sensitivity is advantageous to provide a high signal-to-noise ratio for our measurements and achieve a higher lift-off distance. For our measurements, the photon-shot-noise-limited sensitivity of the diamondbased magnetometer is given by

$$
\eta=\frac{4}{3 \sqrt{3}} \frac{h}{g_{\mathrm{NV}} \mu_{B}} \frac{\Delta v}{C \sqrt{I_{0}}}
$$

where $\Delta v$ is the line width of an NVC resonance, $C$ is the measurement contrast (the reduction in fluorescence

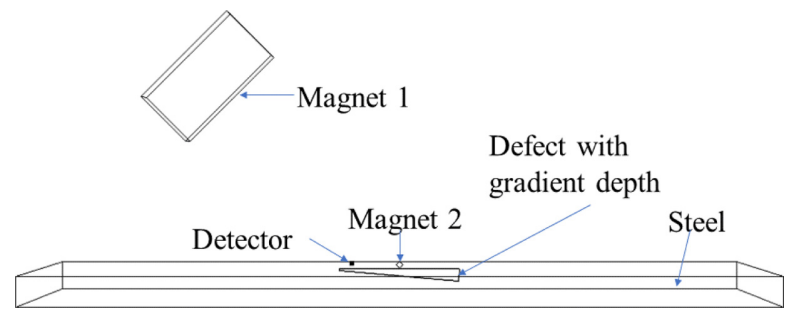

FIG. 10. The setup of the COMSOL simulation: for simplicity, the air space and mesh network have been ignored. The setup shown here is for the gradient-depth sample. The detector here refers to the diamond. 

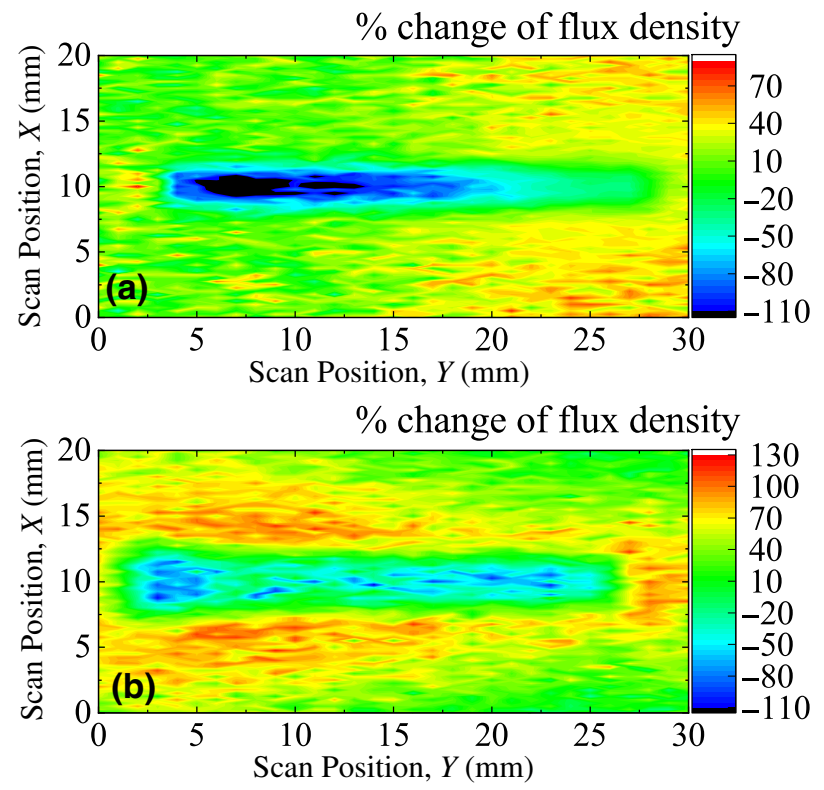

FIG. 11. The 2D scan simulation results on two 316-stainlesssteel samples. (a) sample 1; (b) sample 2.

when on resonance compared to when not on resonance), and $I_{0}$ is the photon collection rate [46]. For our purposes, it is desirable to achieve a high sensitivity with a high dynamic range. To achieve a high dynamic range, a large line width is required; however, this degrades the sensitivity and thus reduces both the signal-to-noise and the achievable lift-off of the sensor from the surface of the material under inspection. In this work, a compromise between the two is found through the use of highly inhomogeneous bias fields near to the diamond with a second magnet to reduce the inhomogeneous bias field incident upon the diamond. The highly inhomogeneous magnetic flux across the active sensing area of the diamond leads to a line width of $13.9 \pm 0.1 \mathrm{MHz}$. Through using the NVC gyromagnetic ratio $(\gamma=28 \mathrm{~Hz} / \mathrm{nT})$, the dynamic range is estimated to be $0.5 \mathrm{mT}$. Significant benefits are expected with the implementation of a proportional integral differential (PID) system that can track the NVC resonance, which would extend the dynamic range while preventing any loss in sensitivity [3].

[1] M. W. Doherty, N. B. Manson, P. Delaney, F. Jelezko, J. Wrachtrup, and L. C. Hollenberg, The nitrogen-vacancy colour centre in diamond, Phys. Rep. 528, 1 (2013).

[2] J. F. Barry, J. M. Schloss, E. Bauch, M. J. Turner, C. A. Hart, L. M. Pham, and R. L. Walsworth, Sensitivity optimization for NV-diamond magnetometry, Rev. Mod. Phys. 92, 015004 (2020).

[3] H. Clevenson, L. M. Pham, C. Teale, K. Johnson, D. Englund, and D. Braje, Robust high-dynamic-range vector magnetometry with nitrogen-vacancy centers in diamond, Appl. Phys. Lett. 112, 252406 (2018).

[4] D. M. Toyli, D. J. Christle, A. Alkauskas, B. B. Buckley, C. G. Van de Walle, and D. D. Awschalom, Measurement and Control of Single Nitrogen-Vacancy Center Spins above 600 K, Phys. Rev. X 2, 031001 (2012).

[5] F. Bachmair, Diamond sensors for future high energy experiments, Nucl. Instrum. Methods A 831, 370 (2016).

[6] G. Balasubramanian, I. Y. Chan, R. Kolesov, M. Al-Hmoud, J. Tisler, C. Shin, C. Kim, A. Wojcik, P. R. Hemmer, A. Krueger, T. Hanke, A. Leitenstorfer, R. Bratschitsch, F. Jelezko, and J. Wrachtrup, Nanoscale imaging magnetometry with diamond spins under ambient conditions, Nature 455, 648 (2008).

[7] J. R. Maze, P. L. Stanwix, J. S. Hodges, S. Hong, J. M. Taylor, P. Cappellaro, L. Jiang, M. V. G. Dutt, E. Togan, A. S. Zibrov, A. Yacoby, R. L. Walsworth, and M. D. Lukin, Nanoscale magnetic sensing with an individual electronic spin in diamond, Nature 455, 644 (2008).

[8] J. M. Taylor, P. Cappellaro, L. Childress, L. Jiang, D. Budker, P. R. Hemmer, A. Yacoby, R. Walsworth, and M. D. Lukin, High-sensitivity diamond magnetometer with nanoscale resolution, Nat. Phys. 4, 810 (2008).

[9] L. M. Pham, D. L. Sage, P. L. Stanwix, T. K. Yeung, D. Glenn, A. Trifonov, P. Cappellaro, P. R. Hemmer, M. D. Lukin, H. Park, A. Yacoby, and R. L. Walsworth, Magnetic field imaging with nitrogen-vacancy ensembles, New J. Phys. 13, 045021 (2011).

[10] L. Rondin, J. P. Tetienne, T. Hingant, J. F. Roch, P. Maletinsky, and V. Jacques, Magnetometry with nitrogenvacancy defects in diamond, Rep. Prog. Phys. 77, 056503 (2014).

[11] J. M. Schloss, J. F. Barry, M. J. Turner, and R. L. Walsworth, Simultaneous Broadband Vector Magnetometry Using Solid-State Spins, Phys. Rev. Appl. 10, 034044 (2018).

[12] H. Clevenson, M. E. Trusheim, C. Teale, T. Schröder, D. Braje, and D. Englund, Broadband magnetometry and temperature sensing with a light-trapping diamond waveguide, Nat. Phys. 11, 393 (2015).

[13] J. F. Barry, M. J. Turner, J. M. Schloss, D. R. Glenn, Y. Song, M. D. Lukin, H. Park, and R. L. Walsworth, Optical magnetic detection of single-neuron action potentials using quantum defects in diamond, Proc. Natl. Acad. Sci. USA 113, 14133 (2016).

[14] J. L. Webb, J. D. Clement, L. Troise, S. Ahmadi, G. J. Johansen, A. Huck, and U. L. Andersen, Nanotesla sensitivity magnetic field sensing using a compact diamond nitrogen-vacancy magnetometer, Appl. Phys. Lett. 114, 231103 (2019).

[15] Y. Xie, H. Yu, Y. Zhu, X. Qin, X. Rong, C.-K. Duan, and J. Du, A hybrid magnetometer towards femtotesla sensitivity under ambient conditions, Science Bulletin (2020).

[16] C. Zhang, F. Shagieva, M. Widmann, M. Kuebler, V. Vorobyov, P. Kapitanova, J. Isoya, and J. Wrachtrup, Diamond Magnetometry and Gradiometry with Subpicotesla DC Sensitivity, arXiv:2012.15706v2 (2021).

[17] T. Wolf, P. Neumann, K. Nakamura, H. Sumiya, T. Ohshima, J. Isoya, and J. Wrachtrup, Subpicotesla Diamond Magnetometry, Phys. Rev. X 5, 041001 (2015). 
[18] D. Le Sage, L. M. Pham, N. Bar-Gill, C. Belthangady, M. D. Lukin, A. Yacoby, and R. L. Walsworth, Efficient photon detection from color centers in a diamond optical waveguide, Phys. Rev. B 85, 121202(R) (2012).

[19] G. Chatzidrosos, A. Wickenbrock, L. Bougas, H. Zheng, O. Tretiak, Y. Yang, and D. Budker, Eddy-Current Imaging with Nitrogen-Vacancy Centers in Diamond, Phys. Rev. Appl. 11, 014060 (2019).

[20] P. Cawley, Non-destructive testing - current capabilities and future directions, Proc. Inst. Mech. Eng. 215, 213 (2001).

[21] Z. Wang, Y. Gu, and Y. Wang, A review of three magnetic NDT technologies, J. Magn. Magn. Mater 324, 382 (2012).

[22] S. Gholizadeh, A review of non-destructive testing methods of composite materials, Proc. Struct. Integrity 1, 50 (2016).

[23] M. P. Papaelias, C. Roberts, and C. L. Davis, A review on non-destructive evaluation of rails: State-of-the-art and future development, Proc. Inst. Mech. Eng. 222, 367 (2008).

[24] S. K. Dwivedi, M. Vishwakarma, and P. Soni, Advances and researches on non destructive testing: A review, Mater. Today: Proc. 5, 3690 (2018). 7th International Conference of Materials Processing and Characterization, March 17-19, 2017.

[25] D. Jiles, Review of magnetic methods for nondestructive evaluation, NDT Int. 21, 311 (1988).

[26] D. Jiles, Review of magnetic methods for nondestructive evaluation (part 2), NDT Int. 23, 83 (1990).

[27] C. Mandache and L. Clapham, A model for magnetic flux leakage signal predictions, J. Phys. D: Appl. Phys. 36, 2427 (2003).

[28] M. Afzal and S. Udpa, Advanced signal processing of magnetic flux leakage data obtained from seamless gas pipeline, NDT E Int. 35, 449 (2002).

[29] K. S. Ryu, D. L. Atherton, and L. Clapham, Effect of pit geometry and bulk stress on near- and far-side calculated MFL signals*, J. Phys. D: Appl. Phys. 35, 2693 (2002).

[30] Y. Shi, C. Zhang, R. Li, M. Cai, and G. Jia, Theory and application of magnetic flux leakage pipeline detection, Sensors 15, 31036 (2015).

[31] Y. Li, J. Wilson, and G. Y. Tian, Experiment and simulation study of 3D magnetic field sensing for magnetic flux leakage defect characterisation, NDT E Int. 40, 179 (2007).

[32] H. Zuoying, Q. Peiwen, and C. Liang, 3D FEM analysis in magnetic flux leakage method, NDT E Int. 39, 61 (2006).

[33] Y. Lijian, L. Gang, Z. Guoguang, and G. Songwei, in 2009 9th International Conference on Electronic Measurement Instruments (2009), p. 2-876.

[34] A. Joshi, L. Udpa, S. Udpa, and A. Tamburrino, Adaptive wavelets for characterizing magnetic flux leakage signals from pipeline inspection, IEEE Trans. Magn. 42, 3168 (2006).

[35] M. R. Kandroodi, F. Shirani, B. N. Araabi, M. N. Ahmadabadi, and M. M. Bassiri, in 2013 9th Asian Control Conference (ASCC) (2013), p. 1.

[36] M. R. Kandroodi, B. N. Araabi, M. N. Ahmadabadi, F. Shirani, and M. M. Bassiri, in 2013 21st Iranian conference on electrical engineering (2013), p. 1.

[37] M. Rostami Kandroodi, B. Nadjar Araabi, M. Mansoob Bassiri, and M. Nili Ahmadabadi, Estimation of depth and length of defects from magnetic flux leakage measurements: Verification with simulations, experiments, and pigging data, IEEE Trans. Magn. 53, 1 (2017).

[38] C.-W. Liang, E. Balaban, E. Ahmad, Z. Zhang, J. Sexton, and M. Missous, A real time high sensitivity high spatial resolution quantum well Hall effect magnetovision camera, Sens. Actuators A: Phys. 265, 127 (2017).

[39] J. M. Watson, C. W. Liang, J. Sexton and M. Missous, Insight 62, 396 (2020).

[40] P. Bevington, R. Gartman, W. Chalupczak, C. Deans, L. Marmugi, and F. Renzoni, Non-destructive structural imaging of steelwork with atomic magnetometers, Appl. Phys. Lett. 113, 1 (2018).

[41] R. L. Patel, L. Q. Zhou, A. C. Frangeskou, G. A. Stimpson, B. G. Breeze, A. Nikitin, M. W. Dale, E. C. Nichols, W. Thornley, B. L. Green, M. E. Newton, A. M. Edmonds, M. L. Markham, D. J. Twitchen, and G. W. Morley, Subnanotesla Magnetometry with a Fiber-Coupled Diamond Sensor, Phys. Rev. Appl. 14, 044058 (2020).

[42] H. A. R. El-Ella, S. Ahmadi, A. M. Wojciechowski, A. Huck, and U. L. Andersen, Optimised frequency modulation for continuous-wave optical magnetic resonance sensing using nitrogen-vacancy ensembles, Opt. Express 25, 14809 (2017).

[43] A. J. Petruska and J. J. Abbott, Optimal permanent-magnet geometries for dipole field approximation, IEEE Trans. Magn. 49, 811 (2013).

[44] L. Q. Zhou, R. L. Patel, A. C. Frangeskou, A. Nikitin, B. L. Green, B. G. Breeze, S. Onoda, J. Isoya, and G. W. Morley, Data for: "Imaging Damage in Steel Using a Diamond Magnetometer," https://wrap.warwick.ac.uk/145914 (2021).

[45] Y. Chu, N. P. De Leon, B. J. Shields, B. Hausmann, R. Evans, E. Togan, M. J. Burek, M. Markham, A. Stacey, A. S. Zibrov, A. Yacoby, D. J. Twitchen, M. Loncar, H. Park, P. Maletinsky, and M. D. Lukin, Coherent optical transitions in implanted nitrogen vacancy centers, Nano Lett. 14, 1982 (2014).

[46] A. Dréau, M. Lesik, L. Rondin, P. Spinicelli, O. Arcizet, J. F. Roch, and V. Jacques, Avoiding power broadening in optically detected magnetic resonance of single NV defects for enhanced dc magnetic field sensitivity, Phys. Rev. B 84, 195204 (2011). 